\title{
A Simple Ultra-Wideband Suspended Stripline Bandpass Filter With Very Wide Stop-Band
}

\author{
Atallah Balalem, Wolfgang Menzel, Fellow, IEEE, Jan Machac, Senior Member, IEEE, and \\ Abbas Omar, Fellow, IEEE
}

\begin{abstract}
This letter introduces a simple ultra-wideband suspended stripline filter with a pass-band from 3.1 to $10.6 \mathrm{GHz}$ and a very wide stop-band up to more than $25 \mathrm{GHz}$. The filter is realized by capactive coupling of a quasi-lumped low-pass filter to the $\mathrm{I} / \mathrm{O}$ ports. Insertion loss in the pass-band is better than $0.5 \mathrm{~dB}$. The filter has a length of $13.8 \mathrm{~mm}$. Excellent agreement is achieved between measured and simulated results.
\end{abstract}

Index Terms-Bandpass filter (BPF), suspended stripline filter, ultra-wideband (UWB) filter.

\section{INTRODUCTION}

$\mathbf{U}$ LTRA-WIDEBAND (UWB) wireless communication systems with 3.1-10.6 GHz frequency band have gained an increased interest in recent years, since they transmit higher data rates with lower transmitted power. Filters represent an essential component of such systems. Various filter structures have been proposed in the literature, e.g., [1]-[7]. The combination of a lowpass filter with a high-pass filter is a classical structure for such kind of filters [1], [2]. It occupies, however, a large area, which may increase the losses of the filter. In [3], an UWB filter was built by cascading various ring filters, while, in [4] and [5], UWB filters were designed using multi-mode resonators. However, due to the long transmission line sections used in these structures, higher order resonances do not allow a wide stop band. An UWB filter introduced in [6] was designed by employing quasi-lumped microstrip resonators built on both sides of the substrate. The fabrication of this filter, however, is difficult due to the via holes necessary to increase the stop-band up to $16 \mathrm{GHz}$.

Due to the structural flexibility of suspended stripline (SSL), including overlapping between the resonators, SSL has been proven as an excellent technique for broadband filter with low losses. Different broadband filters have been achieved including high-pass filters, e.g., [1], [7], [8]. Moreover, using very short transmission line sections (quasi-lumped elements), different filters with wide stop-band can be obtained [8], [9].

\footnotetext{
Manuscript received October 28, 2007; revised November 7, 2007. This work was supported in part by the Czech Ministry of Education, Youth and Sports in the framework of the project "Research in the Area of Prospective Information and Navigation, Technologies" under Grant MSM 6840770014.

A. Balalem and A. Omar are with the Chair of Microwave and, Communication Engineering, University of Magdeburg, Magdeburg 39106, Germany (e-mail: atallah.balalem@ovgu.de; a.omar@ieee.org).

W. Menzel is with the Department of Microwave Techniques, University of Ulm,Ulm D-89069, Germany (e-mail: wolfgang.menzel@uni.ulm.de).

J. Machac is with Faculty of Electrical Engineering, Czech Technical University, Prague, Czech Republic (e-mail: machac@ fel.cvut.cz).

Color versions of one or more of the figures in this letter are available online at http://ieeexplore.ieee.org.

Digital Object Identifier 10.1109/LMWC.2008.916779
}

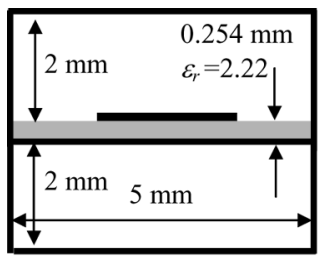

(a)

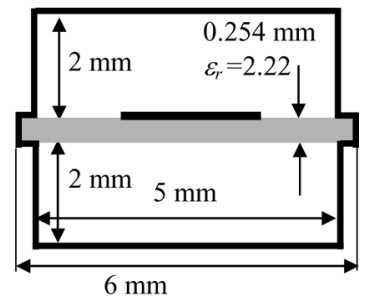

(b)
Fig. 1. Cross-section of SSL (a) used in simulation process, (b) realized in practice.

Since most MICs are microstrip based, there might be a need to integrate the SSL filters with a microstrip. That can be realized by having a grove under the portion of the filter in the carrier block of the circuit and fixing a small cap over the SSL filter to build the channel of the filter, see [10].

In this letter, a simple UWB bandpass filter (BPF) is presented, showing a great simplicity of the design concept compared to earlier known structures. The filter is realized by coupling an SSL quasi-lumped low-pass filter capacitively to the I/O ports. A very wide stop-band is obtained due to the short transmission line sections used in the filter structure. The filter is built on an RT Duroid substrate with a thickness of $0.254 \mathrm{~mm}$ and a relative dielectric constant $\varepsilon_{r}$ of 2.22 . The substrate is shielded in a split-block mount with dimensions of $5 \mathrm{~mm}$ width and $2 \mathrm{~mm}$ height over and below the substrate. The substrate is suspended in the mount by extending it $0.5 \mathrm{~mm}$ inside the mount from both edges (Fig. 1). The additional substrate portion within the clamping region is fully metallized to provide good connection between the mount and the substrate. This is particularly important in case of shunt connections. Due to the small dimensions of the housing mount, the waveguide cut-off frequency is slightly above $20 \mathrm{GHz}$.

\section{Filter Design}

To design the UWB BPF with a frequency range from 3.1 to $10.6 \mathrm{GHz}$ using a low-pass filter, two steps are necessary:

We first designed a low-pass filter with a cut-off frequency of $10.6 \mathrm{GHz}$. In general, the cut-off frequency of the low-pass filter can be adjusted by setting proper values of the lumped elements of the filter [11]. The capacitive and inductive elements of the filter are realized by short sections of high/low-impedance transmission lines. The low impedance sections are implemented by adding a ground metallization at the backside of the substrate [12]. Series inductances are realized by short sections of a high-impedance transmission line on one side of the substrate, 


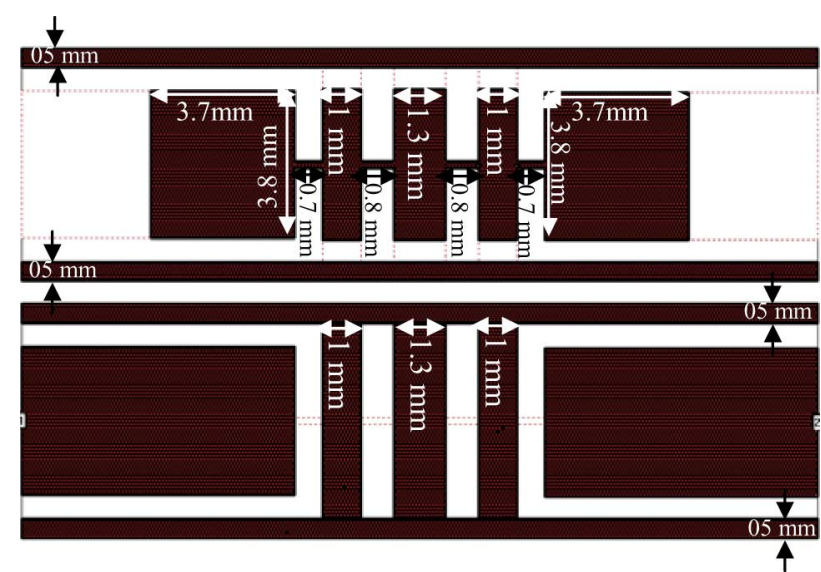

Fig. 2. Top (up) and bottom (down) layouts of the SSL UWB filter using a coupled low-pass filter.

while series capacitances, as used later on, are formed by overlapping wide strips on opposite sides of the substrate. Characteristic impedance $Z_{o}$ and effective dielectric constant $\varepsilon_{\text {eff } f}$ of these transmission lines have been determined using a commercial MoM simulator [13]. The transmission line lengths $l_{k, j}$ of the filter elements, assumed to be much shorter than the wavelength, are calculated from

$$
\begin{aligned}
C_{k} & =\frac{l_{k}}{Z_{o k} \cdot v_{p h, k}} \\
L_{j} & =\frac{l_{j} \cdot Z_{o j}}{v_{p h, j}}
\end{aligned}
$$

where the indices $k$ and $j$ correspond to elements with capacitive and inductive character, respectively. $C_{k}$ and $L_{j}$ are the capacitance and inductance of the equivalent circuit of the filter. The phase velocity is calculated by

$$
v_{p h}=\frac{c}{\sqrt{\varepsilon_{e f f}}} .
$$

Having determined all transmission line lengths the low-pass filter can be combined, however, some optimization can be done to take into account the involved discontinuities and to achieve good matching in the pass-band. The dimensions of the low-pass filter portion are given as part of the overall filter in Fig. 2.

Next, we must suppress transmission in the frequency band below $3.1 \mathrm{GHz}$. The SSL technology provides strong broadside coupling between transmission lines on the opposite sides of the substrate [7], [8]. By overlapping a section of the original input and output lines of the low-pass filter with new input and output lines on the other side of the substrate, capacitive highpass sections [12] are formed to shape the lower filter edge. Fig. 2 shows the top and bottom side layouts of the filter, while Fig. 3 shows the equivalent circuit of the structure.

\section{EXPERIMENTAL RESULTS}

The proposed filter was optimized, fabricated, clamped into the mount, and soldered to coaxial connectors fixed at the ends of the mount (see Fig. 4). Measurements were done using a coaxial calibration; thus the transitions from SSL to coaxial line as well as some portion of SLL transmission line are included into the experimental results. Fig. 5 shows the simulated and measured return and insertion loss of the filter, in addition it

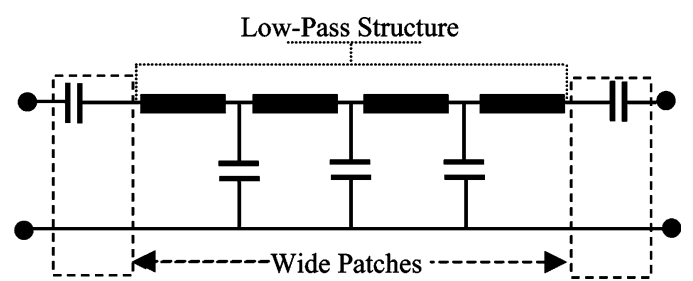

Fig. 3. Equivalent circuit of the UWB BPF using a coupled low-pass filter structure.

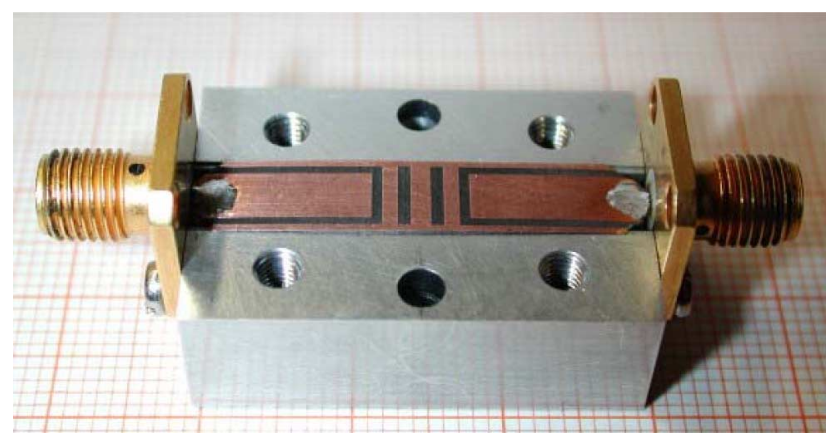

Fig. 4. Photograph of the UWB BPF with opened mount.

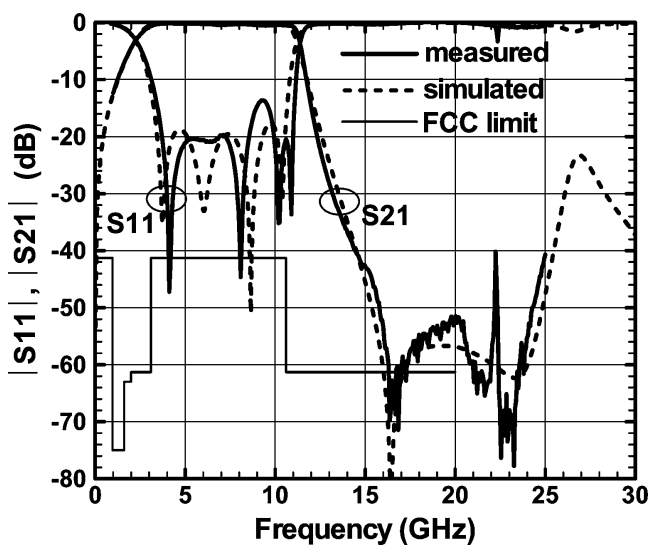

Fig. 5. Simulated and measured insertion and return loss of the UWB filter.

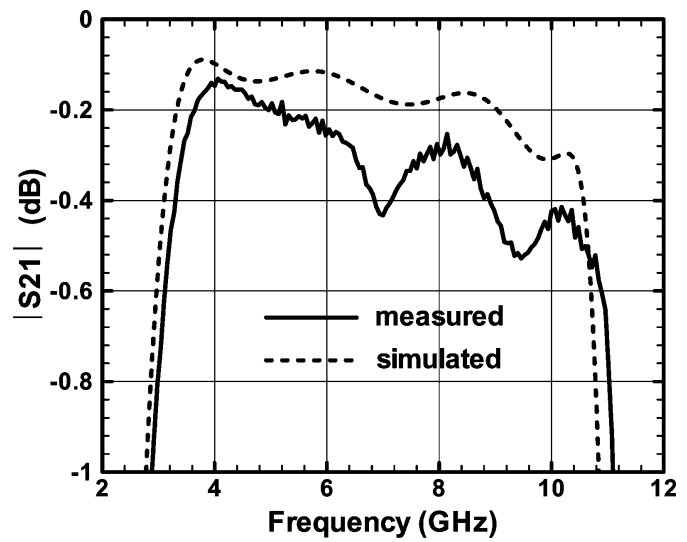

Fig. 6. Simulated and measured insertion loss at the pass-band of the UWB filter.

shows the FCC limit for the hand-held systems [14]. As expected, the filter has a very wide stop-band with a good rejection of better than $40 \mathrm{~dB}$ up to $25 \mathrm{GHz}$. The insertion loss of the filter in the pass-band is better than $0.5 \mathrm{~dB}$ as shown in Fig. 6. Fig. 7 


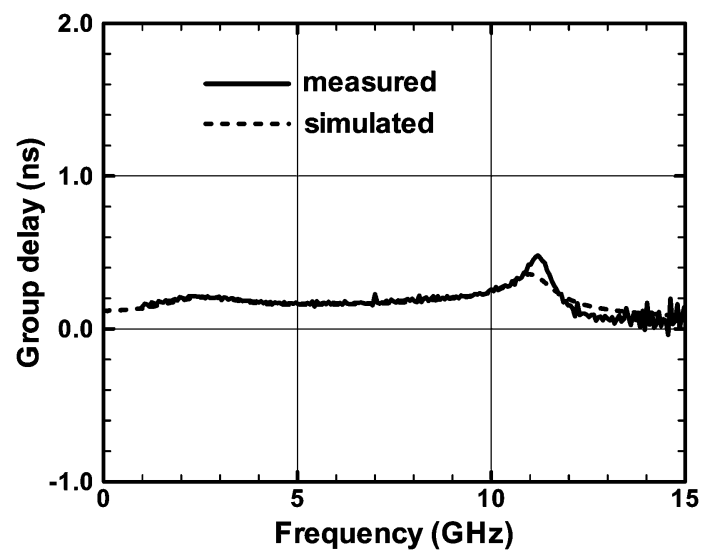

Fig. 7. Measured and simulated group delay of the UWB filter.

shows the group delay of the filter. The group delay variation in the pass-band is smaller than $0.19 \mathrm{~ns}$.

\section{CONCLUSION}

A simpleUWB BPF has been presented. This filter has been designed by capacitively coupling a quasi-lumped suspended stripline low-pass filter to the I/O ports. A very wide stop-band with a rejection higher than $40 \mathrm{~dB}$ up to $25 \mathrm{GHz}$ is achieved. The insertion loss in the passband is better than $0.5 \mathrm{~dB}$. The maximum variation of the filter group delay within the passband is $0.19 \mathrm{~ns}$.

\section{REFERENCES}

[1] W. Menzel, M. S. Tito, and L. Zhu, "Low-loss ultra-wideband (UWB) filters using suspended stripline," in Proc. Asia-Pacific Microw. Conf. APMC., Suzhou, China, Dec. 2005, pp. 2148-2151.
[2] Y. S. Lin, W. C. Ku, C. H. Wang, and C. H. Chin, "Wideband coplanar-waveguide bandpass filters with good stopband rejection," IEEE Microw. Wireless Compon. Lett., vol. 14, no. 9, pp. 422-424, Sep. 2004.

[3] H. Ishida and K. Araki, "Design and analysis of UWB bandpass filter with ring filter," in IEEE MTT-S Int. Dig., Jun. 2004, pp. $1307-1310$.

[4] L. Zhu, S. Sun, and W. Menzel, "Ultra-wideband (UWB) bandpass filter using multiple-mode resonator," IEEE Microw. Wireless Compon. Lett., vol. 15, no. 11, pp. 796-798, Nov. 2005.

[5] H. Wang, L. Zhu, and W. Menzel, "Ultra-wideband bandpass filters with hybrid microstrip/CPW structure," IEEE Microw. Wireless Compon. Lett., vol. 15, no. 12, pp. 844-846, Dec. 2005.

[6] T. N. Kuo, S. C. Lin, and C. H. Chen, "Compact ultra-wideband bandpass filters using composite microstrip-coplanar-waveguide structure," IEEE Trans. Microw. Theory Tech., vol. 54, no. 10, pp. 3772-3778, Oct. 2006.

[7] W. Schwab, F. Boegelsack, and W. Menzel, "Multilayer suspended stripline and coplanar line filters," IEEE Trans. Microw. Theory Tech., vol. 46, no. 7, pp. 1403-1407, Jul. 1994.

[8] W. Menzel and A. Balalem, "Quasi-lumped suspended stripline filters and diplexers," IEEE Trans. Microw. Theory Tech., vol. 53, no. 10, pp. 3230-3237, Oct. 2005.

[9] W. Menzel, "A novel miniature suspended stripline filter," in Proc. Euro. Microw. Conf., Munich, Germany, Oct. 2003, pp. 1047-1050.

[10] W. Menzel and M. S. Tito, "Miniaturized suspended stripline filters for integration into extended circuits," in Proc. Euro. Microw. Conf., Manchester, U.K., Sep. 2006, pp. 909-912.

[11] G. Matthaei, L. Young, and E. M. T. Jones, Microwave Filters, Impedance-Matching and Coupling Structures. Norwood, MA: Artech House, 1980.

[12] W. Menzel, "Broadband filter circuits using extended suspended substrate transmission line configuration," in Proc. 22nd Eur. Microw. Conf., Helsinki, Finland, Oct. 1992, pp. 459-463.

[13] SONNET, Version 10.52. Sonnet Software, Inc.

[14] FCC, "Revision of Part 15 of the Commission's Rules Regarding Ultrawideband Transmission Systems," Federal Communication Commission, Tech. Rep, ET-Docket 98-153, FCC02-48, 2002. 